\section{Distributed Coordinated Tracking With a Dynamic Leader for Multiple Euler-Lagrange Systems}

Jie Mei, Wei Ren, Member, IEEE, and Guangfu Ma

\begin{abstract}
In this note, we study a distributed coordinated tracking problem for multiple networked Euler-Lagrange systems. The objective is for a team of followers modeled by full-actuated Euler-Lagrange equations to track a dynamic leader whose vector of generalized coordinates is time varying under the constraints that the leader is a neighbor of only a subset of the followers and the followers have only local interaction. We consider two cases: i) The leader has a constant vector of generalized coordinate derivatives, and ii) The leader has a varying vector of generalized coordinate derivatives. In the first case, we propose a distributed continuous estimator and an adaptive control law to account for parametric uncertainties. In the second case, we propose a model-independent sliding mode control algorithm. Simulation results on multiple networked two-link revolute joint arms are provided to show the effectiveness of the proposed control algorithms.
\end{abstract}

Index Terms-Cooperative control, coordinated tracking, distributed control, Euler-Lagrange system, multi-agent systems.

\section{INTRODUCTION}

There has been a great interest in distributed control of multi-agent systems due to many potential applications. Examples of interesting research directions include coverage control, consensus, formation control, and flocking (see, e.g., [1]-[3] and references therein). These problems often focus on two cases, namely, the case where there does not exist a leader and the case where there exists a leader. While the leaderless case has some applications such as rendezvous of a group of agents, the presence of a leader can broaden the applications as a group objective can be encapsulated by the leader.

In this note, we are interested in a distributed coordinated tracking problem for multiple networked Euler-Lagrange systems. Here, the objective is that a team of followers modeled by fully-actuated Euler-Lagrange equations tracks a dynamic leader in the sense that the vectors of generalized coordinates of the followers approach that of the dynamic leader. Even fully-actuated Euler-Lagrange models are not as general as underactuated Euler-Lagrange models, they can still be used to represent a class of robot manipulators, fully-actuated autonomous vehicles, and spacecraft. Therefore, distributed control of multiple fully-actuated Euler-Lagrange systems has numerous applications.

Most existing work in distributed control of multi-agent systems focuses on linear systems with first-order or second-order dynamics. We refer the readers to [4], [5] and references therein for more details on leaderless consensus problems. In the case where there exists a leader, with the aid of distributed observers, the authors in [6] study the coordinated tracking problem with an active leader for first-order dynamics.

Manuscript received October 19, 2010; revised January 19, 2011; accepted January 19, 2011. Date of publication January 31, 2011; date of current version June 08, 2011. This work was supported by a National Science Foundation CAREER Award (EECS-0748287) and by the China Scholarship Council. Recommended by Associate Editor J. Cortes.

J. Mei and G. Ma are with the Department of Control Science and Engineering, Harbin Institute of Technology, Heilongjiang 150001, China (e-mail: meij.hit@gmail.com; magf@hit.edu.cn).

W. Ren is with the Department of Electrical and Computer Engineering, Utah State University, Logan, UT 84322 USA (e-mail: wei.ren@ usu.edu).

Color versions of one or more of the figures in this technical note are available online at http://ieeexplore.ieee.org.

Digital Object Identifier 10.1109/TAC.2011.2109437
However, the leader's acceleration is required by all followers to design the distributed observers. Reference [7] proposes a coordinated tracking algorithm with a time-varying leader for first-order dynamics. However, [7] requires the estimates of the neighbors' velocities. In [8], a coordinated tracking problem is studied for first-order dynamics in the presence of time-varying delays. An estimator is proposed to estimate the leader's velocity, but the leader's acceleration is required by all followers. In [9], distributed coordinated tracking algorithms are studied in the absence of velocity or acceleration measurements. Note that the aforementioned references focus on single- or double-integrator linear systems. Although we consider fully-actuated Euler-Lagrange models, the classical method like feedback linearization cannot be used to deal with the problem discussed in this note because there exist parametric uncertainties in the models. Thus, the results for multi-agent systems modeled by single- or double-integrator dynamics cannot be directly applied to our problem.

There has been some recent work on control of multiple Euler-Lagrange systems in both the leaderless case [10], [11] and the case with a leader or equivalently a reference [12]-[16]. The author in [10] proposes three distributed leaderless consensus algorithms for multiple Euler-Lagrange systems. In [11], a controller based on potential functions is proposed for multiple Euler-Lagrange systems to achieve leaderless flocking (i.e., velocity synchronization and collision avoidance). Communication delays and switching network topologies are also considered. The authors in [12] propose a model-independent cross-coupled controller for position synchronization of multi-axis motions. In [13], output synchronization of multiple Euler-Lagrange systems is studied under a passivity-based framework. The authors address both fixed and switching network topologies and consider communication delays in the network. The nonlinear contraction analysis is used in [14] to analyze the stability of cooperative tracking control laws for multiple robotic manipulators. Utilizing potential functions, the authors in [15] design a control scheme that can force multiple robots modeled by fully-actuated Euler-Lagrange equations to move as a group inside a desired region while maintaining a minimum distance among themselves. The robots can finally achieve synchronization on their vectors of generalized coordinate derivatives. Despite the fact that tracking of a dynamic leader whose vector of generalized coordinates is time varying is considered in [12]-[15], it is assumed that all followers have access to the dynamic leader. Unfortunately, this assumption is rather restrictive and not realistic. In [16], the authors study the problem of position synchronization of Euler-Lagrange systems with communication constraints caused by delays and limited data rates, where the leader modeled by fully-actuated Euler-Lagrange equations is a neighbor of only a subset of the followers and the closed-loop system is shown to be input-to-state stable. In the absence of the network effects, the result in [16] guarantees global asymptotic tracking of a stationary leader whose vector of generalized coordinates is constant. However, the result is not applicable to accurate tracking of a dynamic leader under the constraint that the leader is a neighbor of only a subset of the followers.

The contribution of the current note is to solve the distributed coordinated tracking problem for multiple networked Euler-Lagrange systems in the presence of a dynamic leader, where the leader is a neighbor of only a subset of the followers and the followers have only local interaction. Two cases will be considered in the current note: i) The leader has a constant vector of generalized coordinate derivatives; ii) The leader has a varying vector of generalized coordinate derivatives.

Comparison with existing work in the literature: In contrast to the coordinated tracking algorithms for first- and second-order linear dynamics [6]-[9], we consider the nonlinear Euler-Lagrange systems. In 
contrast to the leaderless algorithms for multiple networked Euler-Lagrange systems [10], [11], we deal with coordinated tracking with a dynamic leader. In contrast to the tracking algorithms for multiple networked Euler-Lagrange systems [12]-[15], where all followers have access to the dynamic leader, and the input-to-state framework in [16], the dynamic leader in the current note is a neighbor of only a subset of the followers. A preliminary version of the note has appeared in [17].

\section{BACKGROUND AND PROBLEM STATEMENT}

Suppose that there exist $n$ followers, labeled as agents or followers 1 to $n$, and a leader labeled as agent 0 , in a team. The $n$ followers are represented by Euler-Lagrange equations of the form

$$
M_{i}\left(q_{i}\right) \ddot{q}_{i}+C_{i}\left(q_{i}, \dot{q}_{i}\right) \dot{q}_{i}+g_{i}\left(q_{i}\right)=\tau_{i}, \quad i=1, \cdots, n
$$

where $q_{i} \in \mathbb{R}^{p}$ is the vector of generalized coordinates, $M_{i}\left(q_{i}\right) \in$ $\mathbb{R}^{p \times p}$ is the symmetric positive definite inertia matrix, $C_{i}\left(q_{i}, \dot{q}_{i}\right) \dot{q}_{i} \in$ $\mathbb{R}^{p}$ is the vector of Coriolis and centrifugal forces, $g_{i}\left(q_{i}\right)$ is the vector of gravitational force, and $\tau_{i} \in \mathbb{R}^{p}$ is the vector of control force on the $i$ th agent. The leader's vector of generalized coordinates and vector of generalized coordinate derivatives are denoted by, respectively, $q_{0}$ and $\dot{q}_{0}$. Throughout the subsequent analysis we shall assume that the dynamics satisfy the following assumptions [18]:

(A1) Boundedness: For any $i$, there exist positive constants $k_{\underline{m}}$, $k_{\bar{m}}, k_{C}$, and $k_{g_{i}}$ such that $0<k_{\underline{m}} I_{p} \leq M_{i}\left(q_{i}\right) \leq k_{\bar{m}} I_{p}$, where $I_{p}$ denotes the $p \times p$ identity matrix, $\left\|C_{i}(x, y) z\right\| \leq k_{C}\|y\|\|z\|$ for all vectors $x, y, z \in \mathbb{R}^{p}$, and $\left\|g_{i}\left(q_{i}\right)\right\| \leq k_{g_{i}}$. Hereafter, we use $\|\cdot\|$ to denote the Euclidean norm.

(A2) Skew symmetry property: $\dot{M}_{i}\left(q_{i}\right)-2 C_{i}\left(q_{i}, \dot{q}_{i}\right)$ is skew symmetric.

(A3) Linearity in the parameters: $M_{i}\left(q_{i}\right) x+C_{i}\left(q_{i}, \dot{q}\right) y+g_{i}\left(q_{i}\right)=$ $Y_{i}\left(q_{i}, \dot{q}_{i}, x, y\right) \Theta_{i}$ for all vectors $x, y \in \mathbb{R}^{p}$, where $Y_{i}\left(q_{i}, \dot{q}_{i}, x, y\right)$ is the regressor and $\Theta_{i}$ is a vector for unknown but constant parameters associated with the $i$ th agent.

Let $\mathcal{G} \triangleq(\mathcal{V}, \mathcal{E})$ be a graph of order $k$ with the node set $\mathcal{V} \triangleq$ $\{1, \ldots, k\}$ and the edge set $\mathcal{E} \subseteq \mathcal{V} \times \mathcal{V}$. Here node $i$ denotes the $i$ th agent. In a directed graph, an edge $(i, j) \in \mathcal{E}$ denotes that agent $j$ can receive information from agent $i$ but not vice versa. Here agent $i$ is a neighbor of agent $j$. A directed path from node $i$ to node $j$ is a sequence of edges in a directed graph. If there is a path between every pair of nodes in a graph, then the graph is connected. In an undirected graph, $(i, j) \in \mathcal{E} \Leftrightarrow(j, i) \in \mathcal{E}$. Let the adjacency matrix $\mathcal{A}=\left[a_{i j}\right] \in \mathbb{R}^{k \times k}$ with $a_{i j}>0$ if $(j, i) \in \mathcal{E}$, and $a_{i j}=0$ otherwise. We assume $a_{i i}=0$. For an undirected graph, we assume $a_{i j}=a_{j i}$. The Laplacian matrix $\mathcal{L}=\left[l_{i j}\right] \in \mathbb{R}^{k \times k}$ is defined as $l_{i i}=\sum_{j=1, j \neq i}^{n} a_{i j}$ and $l_{i j}=-a_{i j}, i \neq j$.

Lemma 2.1: [1] Let $\mathcal{G}_{F} \triangleq\left(\mathcal{V}_{F}, \mathcal{E}_{F}\right)$ be the directed graph characterizing the interaction among the $n$ followers with the associated Laplacian matrix $\mathcal{L}_{F}, \overline{\mathcal{G}} \triangleq(\overline{\mathcal{V}}, \overline{\mathcal{E}})$ be the directed graph characterizing the interaction among the leader and the $n$ followers corresponding to $\mathcal{G}_{F}$, and $a_{i 0}>0$ if the leader is a neighbor of follower $i$ and $a_{i 0}=0$ otherwise. If in $\overline{\mathcal{G}}$ the leader has directed paths to all followers, then the matrix $H \triangleq \mathcal{L}_{F}+\operatorname{diag}\left(a_{10}, \cdots, a_{k 0}\right)$ is positive stable. In addition, if $\mathcal{G}_{F}$ is undirected, then $H$ is symmetric positive definite.

Define $q \triangleq\left[q_{1}^{T}, \ldots, q_{n}^{T}\right]^{T}$ and $\dot{q} \triangleq\left[\dot{q}_{1}^{T}, \ldots, \dot{q}_{n}^{T}\right]^{T}$. Also define $M(q) \quad \triangleq \quad \operatorname{diag}\left[M_{1}\left(q_{1}\right), \cdots, M_{n}\left(q_{n}\right)\right]$, $C(q, \dot{q}) \quad \triangleq \quad \operatorname{diag}\left[C_{1}\left(q_{1}, \dot{q}_{1}\right), \cdots, C_{n}\left(q_{n}, \dot{q}_{n}\right)\right], \quad$ and $g(q) \triangleq\left[g_{1}^{T}\left(q_{1}\right), \ldots, g_{n}^{T}\left(q_{n}\right)\right]^{T}$. Let $\mathbf{1}_{n}$ denote the $n \times 1$ column vector of all ones and $\mathbf{0}_{n}$ denote the $n \times 1$ column vector of all zeros. Let $\lambda_{\max }(\cdot)$ and $\lambda_{\min }(\cdot)$ denote, respectively, the maximal and minimum eigenvalue of a symmetric positive definite real matrix.

\section{CoOrdinated TRACKING When THE Leader's Vector of GENERALIZED COORDINATE DERIVATIVES IS CONSTANT}

In this section, we assume that $\dot{q}_{0}$ is constant. The objective here is to design distributed coordinated tracking algorithms for (1) such that $\left\|q_{i}(t)-q_{0}(t)\right\| \rightarrow 0$ and $\dot{q}_{i}(t) \rightarrow \dot{q}_{0}$ as $t \rightarrow \infty$. Before moving on, we introduce the following auxiliary variables:

$$
\begin{gathered}
\dot{q}_{r i} \triangleq \hat{v}_{i}-\alpha \sum_{j=0}^{n} a_{i j}\left(q_{i}-q_{j}\right), \\
s_{i} \triangleq \dot{q}_{i}-\dot{q}_{r i}=\dot{q}_{i}-\hat{v}_{i}+\alpha \sum_{j=0}^{n} a_{i j}\left(q_{i}-q_{j}\right), \\
\quad i=1, \ldots, n
\end{gathered}
$$

where $\alpha$ is a positive constant, $\hat{v}_{i}$ is the $i$ th follower's estimate of the leader's vector of generalized coordination derivatives, $a_{i j}, i, j=$ $1, \ldots, n$, is the $(i, j)$ th entry of the adjacency matrix $\mathcal{A}$ associated with the undirected graph $\mathcal{G}_{A} \triangleq\left(\mathcal{V}, \mathcal{E}_{A}\right)$ characterizing the interaction among the $n$ followers for $q_{i}$ (and $\dot{q}_{i}$ as shown later on), and $a_{i 0}>0$ if the follower $i$ has access to $q_{0}$ (and $\dot{q}_{0}$ as shown later on) and $a_{i 0}=0$ otherwise. From Assumption (A3), we get that

$M_{i}\left(q_{i}\right) \ddot{q}_{r i}+C_{i}\left(q_{i}, \dot{q}_{i}\right) \dot{q}_{r i}+g_{i}\left(q_{i}\right)=Y_{i}\left(q_{i}, \dot{q}_{i}, \ddot{q}_{r i}, \dot{q}_{r i}\right) \Theta_{i}$,

$$
i=1, \ldots, n \text {. }
$$

We propose the following coordinated tracking algorithm for (1) in the presence of parametric uncertainties:

$$
\begin{aligned}
\tau_{i} & =-K_{i} s_{i}-\eta\left(\dot{q}_{i}-\hat{v}_{i}\right)+Y_{i} \widehat{\Theta}_{i} \\
\dot{\hat{v}}_{i} & =-\sum_{j=1}^{n} b_{i j}\left(\hat{v}_{i}-\hat{v}_{j}\right)-b_{i 0}\left(\hat{v}_{i}-\dot{q}_{0}\right), \quad i=1, \ldots, n
\end{aligned}
$$

where $K_{i}$ is a symmetric positive-definite matrix, $\eta$ is a positive constant, $Y_{i} \triangleq Y_{i}\left(q_{i}, \dot{q}_{i}, \ddot{q}_{r i}, \dot{q}_{r i}\right), \widehat{\Theta}_{i}$ is the estimate of $\Theta_{i}, b_{i j}, i, j=$ $1, \ldots, n$, is the $(i, j)$ th entry of the adjacency matrix $\mathcal{B}$ associated with the directed graph $\mathcal{G}_{B} \triangleq\left(\mathcal{V}, \mathcal{E}_{B}\right)$ characterizing the interaction among the $n$ followers for $\hat{v}_{i}$, and $b_{i 0}>0$ if in (4b) follower $i$ has access to $\dot{q}_{0}$ and $b_{i 0}=0$ otherwise. Here $\widehat{\Theta}_{i}$ is updated by the following adaptation law:

$$
\dot{\widehat{\Theta}}_{i}=-\Lambda_{i} Y_{i}^{T} s_{i}, \quad i=1, \ldots, n
$$

where $\Lambda_{i}$ is a symmetric positive-definite matrix. Let $\widetilde{\Theta}_{i} \triangleq \Theta_{i}-\widehat{\Theta}_{i}$, and $\widetilde{\Theta}, \Theta, \widehat{\Theta}, s, \tilde{q}$, and $\hat{v}$ be, respectively, the column stack vectors of $\widetilde{\Theta}_{i}, \Theta_{i}, \widehat{\Theta}_{i}, s_{i}, \tilde{q}_{i} \triangleq q_{i}-q_{0}$, and $\hat{v}_{i}, i=1, \ldots, n$. Hence, the closed-loop system (1) using (4a) can be written in a vector form as

$$
M(q) \dot{s}=-C(q, \dot{q}) s-K s-\eta\left[s-\alpha\left(H_{A} \otimes I_{p}\right) \tilde{q}\right]-Y \widetilde{\Theta}
$$

where $H_{A} \triangleq \mathcal{L}_{A}+\operatorname{diag}\left(a_{10}, \cdots, a_{n 0}\right), Y \triangleq \operatorname{diag}\left(Y_{1}, \cdots, Y_{n}\right)$, and $K \triangleq \operatorname{diag}\left(K_{1}, \cdots, K_{n}\right)$.

Let $\overline{\mathcal{G}}_{A}$ (respectively, $\overline{\mathcal{G}}_{B}$ ) be the directed graph characterizing the interaction among the leader and the followers corresponding to $\mathcal{G}_{A}$ (respectively, $\mathcal{G}_{B}$ ). Before presenting our main results, we need the following lemmas.

Lemma 3.1: If in $\overline{\mathcal{G}}_{B}$ the leader has directed paths to all followers, using (4b), $\hat{v}_{i}(t) \rightarrow \dot{q}_{0}$ exponentially as $t \rightarrow \infty$.

Proof: Let $\bar{v}_{i} \triangleq \hat{v}_{i}-\dot{q}_{0}$ and $\bar{v} \triangleq\left[\bar{v}_{1}^{T}, \ldots, \bar{v}_{n}^{T}\right]^{T}$. Note that $\dot{q}_{0}$ is constant. Then (4b) can be written as $\dot{\bar{v}}_{i}=-\sum_{j=0}^{n} b_{i j}\left(\bar{v}_{i}-\bar{v}_{j}\right)$, which can be written in a vector form as

$$
\dot{\bar{v}}=-\left(\mathcal{L}_{B} \otimes I_{p}\right) \bar{v}-\left[\operatorname{diag}\left(b_{10}, \cdots, b_{n 0}\right) \otimes I_{p}\right] \bar{v}=-\left(H_{B} \otimes I_{p}\right) \bar{v}
$$


where $\mathcal{L}_{B}$ is the Laplacian matrix associated with $\mathcal{B}$ and hence $\mathcal{G}_{B}$, and $H_{B} \triangleq \mathcal{L}_{B}+\operatorname{diag}\left(b_{10}, \cdots, b_{n 0}\right)$.

Because in $\overline{\mathcal{G}}_{B}$ the leader has directed paths to all followers, we conclude from Lemma 2.1 that $H_{B}$ is positive stable. Thus, there exists a symmetric positive definite matrix $P \in \mathbb{R}^{n \times n}$ such that $S \triangleq P H_{B}+$ $H_{B}^{T} P$ is symmetric positive definite. Consider the Lyapunov function $V_{0}=\bar{v}^{T}\left(P \otimes I_{p}\right) \bar{v}$. We can calculate that $\dot{V}_{0}=-\bar{v}^{T}\left(S \otimes I_{p}\right) \bar{v}$. After some manipulation, we can easily get that

$$
\|\bar{v}(t)\| \leq \gamma_{1}\|\bar{v}(0)\| e^{-\gamma_{2} t}
$$

where $\gamma_{1} \triangleq \sqrt{\lambda_{\max }(P) / \lambda_{\min }(P)}, \gamma_{2} \triangleq \lambda_{\min }(S) / 2 \lambda_{\max }(P)$. Therefore, $\bar{v}=0$ is globally exponentially stable, which implies that $\hat{v}_{i}(t) \rightarrow \dot{q}_{0}$ exponentially as $t \rightarrow \infty$.

Lemma 3.2: [19] Consider the following cascade system

$$
\begin{aligned}
& \dot{x}=f(t, x)+h(x, \xi), \quad f(t, 0)=0, h(x, 0)=0, \\
& \dot{\xi}=A \xi, \quad(x, \xi) \in \mathbb{R}^{n} \times \mathbb{R}^{m}
\end{aligned}
$$

where $f(t, x)$ is continuously differentiable in $(t, x)$, and $h(x, \xi)$ is locally Lipschitz in $(x, \xi)$. When $\xi=0,(9)$ can be written as

$$
\dot{x}=f(t, x) \text {. }
$$

If (11) has the origin as a globally asymptotically stable equlibrium, $A$ is Hurwitz, and all solutions of (9) and (10) are bounded, then the cascade system is globally asymptotically stable at the origin.

We have the following theorem with a constant $\dot{q}_{0}$.

Theorem 3.1: Consider the closed-loop system (6). If $\mathcal{G}_{A}$ is undirected and $\mathcal{G}_{B}$ is directed, and in both $\overline{\mathcal{G}}_{A}$ and $\overline{\mathcal{G}}_{B}$ the leader has directed paths to all followers, $\left\|q_{i}(t)-q_{0}(t)\right\| \rightarrow 0$ and $\dot{q}_{i}(t) \rightarrow \dot{q}_{0}$, $i=1, \ldots, n$, as $t \rightarrow \infty$ in the presence of parametric uncertainties.

Proof: Let $x_{1} \triangleq \tilde{q}, x_{2} \triangleq s, x \triangleq\left[x_{1}^{T}, x_{2}^{T}\right]^{T}$, and $\xi \triangleq \hat{v}-\mathbf{1}_{n} \otimes \dot{q}_{0}$. Equations (6) and (4b) can be written as

$$
\begin{aligned}
\dot{x}= & \underbrace{\left[\begin{array}{c}
x_{2}-\alpha\left(H_{A} \otimes I_{p}\right) x_{1} \\
-M^{-1}(q)\left[-\eta \alpha\left(H_{A} \otimes I_{p}\right) x_{1}+Q x_{2}+Y \widetilde{\Theta}\right]
\end{array}\right]}_{f(t, x)} \\
& +\underbrace{\left[\begin{array}{c}
\xi \\
\mathbf{0}_{n p}
\end{array}\right]}_{h(x, \xi)}, \\
\dot{\xi}= & \underbrace{-\left(H_{B} \otimes I_{p}\right)}_{A} \xi
\end{aligned}
$$

where $Q \triangleq C(q, \dot{q})+K+\eta I_{n} \otimes I_{p}$ and $H_{B}$ is defined as in (7). Note that $q=x_{1}+\mathbf{1}_{n} \otimes q_{0}$ and that $\dot{q}$ and $\widetilde{\Theta}$ in (12) are not treated as states, but as functions of $t$. Hence, (12) and (13) takes in the form of the cascade system (9) and (10), and

$$
\dot{x}=\left[\begin{array}{c}
x_{2}-\alpha\left(H_{A} \otimes I_{p}\right) x_{1} \\
-M^{-1}(q)\left[-\eta \alpha\left(H_{A} \otimes I_{p}\right) x_{1}+Q x_{2}+Y \widetilde{\Theta}\right]
\end{array}\right]
$$

takes in the form of (11).

First, we will show that all solutions of (12) and (13) are bounded. Because $\mathcal{G}_{A}$ is undirected and $\mathcal{G}_{B}$ is directed, and in both $\overline{\mathcal{G}}_{A}$ and $\overline{\mathcal{G}}_{B}$ the leader has directed paths to all followers, we conclude from Lemma 2.1 that $H_{A}$ is symmetric positive definite and $H_{B}$ is positive stable. We get that the solution of (13) (i.e., $\xi$ ) is bounded. Consider a nonnegative scalar function as $V(t, x)=(\eta \alpha / 2) x_{1}^{T}\left(H_{A} \otimes I_{p}\right) x_{1}+$
$(1 / 2) x_{2}^{T} M(q) x_{2}+(1 / 2) \widetilde{\Theta}^{T} \Xi \widetilde{\Theta}$, where $\Xi \triangleq \operatorname{diag}\left(\Lambda_{1}^{-1}, \cdots, \Lambda_{n}^{-1}\right)$ is symmetric positive definite. We have that

$$
\begin{aligned}
V & \geq \frac{\eta \alpha}{2} \lambda_{\min }\left(H_{A}\right)\left\|x_{1}\right\|^{2}+\frac{1}{2} k_{\underline{m}}\left\|x_{2}\right\|^{2} \\
& \geq \frac{1}{2} \min \left[\eta \alpha \lambda_{\min }\left(H_{A}\right), k_{\underline{m}}\right]\|x\|^{2}
\end{aligned}
$$

and

$$
\begin{aligned}
\left\|\frac{\partial V}{\partial x}\right\| & =\left\|\left\{\eta \alpha\left[\left(H_{A} \otimes I_{p}\right) x_{1}\right]^{T},\left[M(q) x_{2}\right]^{T}\right\}^{T}\right\|, \\
& \leq \max \left[\eta \alpha \lambda_{\max }\left(H_{A}\right), k_{\bar{m}}\right]\|x\| \\
& \leq \gamma \sqrt{V}
\end{aligned}
$$

where $\gamma \quad \triangleq \quad\left(\sqrt{2} \max \left[\eta \alpha \lambda_{\max }\left(H_{A}\right), k_{\bar{m}}\right]\right) /$ ( $\sqrt{\min \left[\eta \alpha \lambda_{\min }\left(H_{A}\right), k_{\underline{m}}\right.}$. The derivative of $V$ along (14) is

$$
\begin{aligned}
\dot{V}_{(14)}= & \frac{\partial V}{\partial t}+\frac{\partial V}{\partial x} f(t, x) \\
= & -\eta\left[x_{2}-\alpha\left(H_{A} \otimes I_{p}\right) x_{1}\right]^{T}\left[x_{2}-\alpha\left(H_{A} \otimes I_{p}\right) x_{1}\right] \\
& -x_{2}^{T} K x_{2}
\end{aligned}
$$

where we have used Assumption (A2) and the fact that $\dot{\widetilde{\Theta}}=\Xi^{-1} Y^{T} s$ according to (5) and $s \equiv x_{2}$ to obtain the last equality. Note that $\dot{V}_{(14)} \leq 0$ because $\eta>0$ and $K$ is symmetric positive definite. Note from (12) that $\|h(x, \xi)\| \leq\|\xi\|$. Then the derivative of $V$ along (12) can be written as

$$
\dot{V}_{(12)}=\dot{V}_{(14)}+\frac{\partial V}{\partial x} h(x, \xi) \leq\left\|\frac{\partial V}{\partial x}\right\|\|h(x, \xi)\| \leq \gamma\|\xi\| \sqrt{V} .
$$

From (8), noting that $\xi \equiv \bar{v}$, we can get that

$$
\int_{0}^{t}\|\xi(\tau)\| d \tau \leq \gamma_{1}\|\xi(0)\| \int_{0}^{t} e^{-\gamma_{2} \tau} d \tau=\frac{\gamma_{1}\|\xi(0)\|}{\gamma_{2}}\left(1-e^{-\gamma_{2} t}\right) .
$$

After some manipulation, we obtain from (16) and (17) that

$$
\begin{aligned}
\sqrt{V(t, x(t))} & \leq \sqrt{V(0, x(0))}+\frac{\gamma}{2} \int_{0}^{t}\|\xi(\tau)\| d \tau \\
& \leq \sqrt{V(0, x(0))}+\frac{\gamma \gamma_{1}\|\xi(0)\|}{2 \gamma_{2}} .
\end{aligned}
$$

From (18), we can conclude that $V(t, x)$ is uniformly bounded along the solution of (12). It thus follows that the solution of (12) (i.e., $x_{1}$ and $x_{2}$ ) is bounded.

Second, we show that (14) is globally asymptotically stable at the origin. By applying Assumption (A1), and using the above arguments, we can show that $\ddot{V}_{(14)}$ is bounded, which implies that $\dot{V}_{(14)}$ is uniformly continuous. According Barbalat's Lemma [20], we conclude that $\dot{V}_{(14)}(t) \rightarrow 0$ as $t \rightarrow \infty$. Then from (15) we can get that $x_{2}(t)-$ $\alpha\left(H_{A} \otimes I_{p}\right) x_{1}(t) \rightarrow 0$ and $x_{2}(t) \rightarrow 0$ as $t \rightarrow \infty$, which means that $\left(H_{A} \otimes I_{p}\right) x_{1}(t) \rightarrow 0$ as $t \rightarrow \infty$. Because $H_{A}$ is symmetric positive definite, it follows that $x_{1}(t) \rightarrow 0$ as $t \rightarrow \infty$. Note that $V$ is radially unbounded with respect to $x$, it follows that the system (14) is globally asymptotically stable at the origin.

Third, note that $H_{B}$ is positive stable, which implies that $A$ in (13) is Hurwitz. We conclude from Lemma 3.2 that the cascade system (12) and (13) is globally asymptotically stable at the origin, i.e., $x_{1}(t) \rightarrow 0$, $x_{2}(t) \rightarrow 0$ and $\xi(t) \rightarrow 0$ as $t \rightarrow \infty$. Note that $x_{1}=q-\mathbf{1}_{n} \otimes q_{0}$. We can get that $\left\|q_{i}(t)-q_{0}(t)\right\| \rightarrow 0, i=1, \ldots, n$, as $t \rightarrow \infty$. Also note that $x_{2}=\dot{q}-\mathbf{1}_{n} \otimes \dot{q}_{0}-\xi+\alpha\left(H_{A} \otimes I_{p}\right) x_{1}$. We can conclude that $\dot{q}_{i}(t) \rightarrow \dot{q}_{0}, i=1, \ldots, n$, as $t \rightarrow \infty$ because $x_{1}(t) \rightarrow 0, x_{2}(t) \rightarrow 0$, and $\xi(t) \rightarrow 0$ as $t \rightarrow \infty$. 


\section{COORDinated TRACKING WHEN THE LEADER'S VeCTOR OF GENERALIZED COORDINATE DERIVATIVES IS VARYING}

In this subsection, $\dot{q}_{0}(t)$ is allowed to be varying. The objective here is to design a distributed model-independent sliding-mode algorithm for (1) such that $\left\|q_{i}(t)-q_{0}(t)\right\| \rightarrow 0$ and $\left\|\dot{q}_{i}(t)-\dot{q}_{0}(t)\right\| \rightarrow 0$ as $t \rightarrow \infty$. Define the auxiliary variables $s_{i} \triangleq \dot{q}_{i}+\lambda q_{i}, i=0,1, \ldots, n$, where $\lambda$ is a positive constant. Also define the error variable between $s_{i}$ and $s_{0}$ as

$$
\tilde{s}_{i} \triangleq s_{i}-s_{0}=\dot{q}_{i}-\dot{q}_{0}+\lambda\left(q_{i}-q_{0}\right), \quad i=1, \ldots, n .
$$

Then (1) can be written as

$$
M_{i}\left(q_{i}\right) \dot{s}_{i}+C_{i}\left(q_{i}, \dot{q}_{i}\right) s_{i}=\tau_{i}+\lambda M_{i}\left(q_{i}\right) \dot{q}_{i}+\lambda C_{i}\left(q_{i}, \dot{q}_{i}\right) q_{i}-g_{i}\left(q_{i}\right) .
$$

We propose the distributed coordinated tracking algorithm for (1) as

$$
\begin{aligned}
& \tau_{i}=-\alpha {\left[\sum_{j=0}^{n} a_{i j}\left(s_{i}-s_{j}\right)\right] } \\
&-\beta\left(\sum _ { j = 1 } ^ { n } a _ { i j } \left\{\operatorname{sgn}\left[\sum_{k=0}^{n} a_{i k}\left(s_{i}-s_{k}\right)\right]\right.\right. \\
&\left.-\operatorname{sgn}\left[\sum_{k=0}^{n} a_{j k}\left(s_{j}-s_{k}\right)\right]\right\} \\
&\left.+a_{i 0} \operatorname{sgn}\left[\sum_{j=0}^{n} a_{i j}\left(s_{i}-s_{j}\right)\right]\right)
\end{aligned}
$$

where $\alpha$ is a nonnegative constant, $\beta$ is a positive constant, $a_{i j}, i=$ $1, \ldots, n, j=0, \ldots, n$ is defined as in (2), and $\operatorname{sgn}(\cdot)$ is defined componentwise. Note that the right-hand side of (21) is discontinuous. Therefore, the solution is investigated in terms of differential inclusions using nonsmooth analysis [21]-[23]. Because the signum function is measurable and locally essentially bounded, the Filippov solutions for the closed-loop system (1) using (21) exist [21]. Let $\tilde{q}$ be defined as in Section III. Also let $s$ and $\tilde{s}$ be, respectively, the column stack vectors of $s_{i}$ and $\tilde{s}_{i}, i=1, \ldots, n$. We can rewrite the closed-loop system (1) using (21) in a vector form as

$$
\begin{aligned}
\dot{\tilde{s}} \in^{a . e .} \mathcal{K}\left[M ^ { - 1 } ( q ) \left[-C(q, \dot{q}) \tilde{s}-\beta\left(H_{A} \otimes I_{p}\right)\right.\right. \\
\left.\left.\times \operatorname{sgn}\left[\left(H_{A} \otimes I_{p}\right) \tilde{s}\right]-\alpha\left(H_{A} \otimes I_{p}\right) \tilde{s}+\Delta\right]\right]
\end{aligned}
$$

where $\mathcal{K}[\cdot]$ is the differential inclusion, a.e. stands for "almost everywhere," $\Delta \triangleq-M(q)\left(\mathbf{1}_{n} \otimes \dot{s}_{0}\right)-C(q, \dot{q})\left(\mathbf{1}_{p} \otimes s_{0}\right)+$ $\lambda M(q) \dot{q}+\lambda C(q, \dot{q}) q-g(q)$, and $H_{A}$ is defined as in (6). Note that $H_{A}$ is symmetric positive semidefinite because $\mathcal{G}_{A}$ is undirected.

Before moving on, we need the following assumption:

(A4) Both $\dot{q}_{0}$ and $\ddot{q}_{0}$ are bounded, and in particular, $\left\|\mathbf{1}_{n} \otimes \dot{q}_{0}\right\| \leq$ $k_{v}$ and $\left\|\mathbf{1}_{n} \otimes \ddot{q}_{0}\right\| \leq k_{a}$.

Remark 4.1: We do not restrict $q_{0}$ to be bounded in (A4). Most desired trajectories have the properties of (A4), so (A4) is a reasonable assumption. In the control algorithm (21), there is no need to know the value of $\ddot{q}_{0}$.

Next, we show the boundedness of $\Delta$ in (22). From Assumption (A1), there exists $k_{g}$ such that $\|g(q)\| \leq k_{g}$. Following Assumptions (A1) and (A4), we have that

$$
\begin{aligned}
\|\Delta\|= & \|-M(q)\left(\mathbf{1}_{n} \otimes \ddot{q}_{0}\right)-C(q, \dot{q})\left(\mathbf{1}_{n} \otimes \dot{q}_{0}\right) \\
& +\lambda M(q) \dot{\tilde{q}}+\lambda C(q, \dot{q}) \tilde{q}-g(q) \| \\
\leq & k_{\bar{m}} k_{a}+k_{C} k_{v}\|\dot{q}\|+\lambda k_{\bar{m}}\|\dot{\tilde{q}}\| \\
& +\lambda k_{C}\|\dot{q}\|\|\tilde{q}\|+k_{g} .
\end{aligned}
$$

Note that (19) can be written in a vector form as $\tilde{s}=\dot{\tilde{q}}+\lambda \tilde{q}$. Multiplying $e^{\lambda \tau}$ on both sides and integrating from 0 to $t$, we have that

$$
\tilde{q}(t)=e^{-\lambda t}\left[\tilde{q}(0)+\int_{0}^{t} e^{\lambda \tau} \tilde{s}(\tau) d \tau\right]
$$

From (24), we have that

$$
\|\tilde{q}(t)\| \leq e^{-\lambda t}\|\tilde{q}(0)\|+\frac{\sup _{0 \leq \tau \leq t}\|\tilde{s}(\tau)\|}{\lambda}\left(1-e^{-\lambda t}\right) .
$$

Define a norm-like function $\|x\|_{M} \triangleq \sqrt{x^{T} M(q) x}$, where $x \in \mathbb{R}^{n p}$. Then $\sqrt{k_{\underline{m}}}\|x\| \leq\|x\|_{M} \leq \sqrt{k_{\bar{m}}}\|x\|$ for all $t \geq 0$. We can get that $\|\tilde{s}(t)\| \leq\|\tilde{s}(t)\|_{M} / \sqrt{k_{\underline{m}}}$ for all $t \geq 0$. It thus follows that $\sup _{0 \leq \tau \leq t}\|\tilde{s}(\tau)\| \leq \sup _{0 \leq \tau \leq t}\|\tilde{s}(\tau)\|_{M} / \sqrt{k_{\underline{m}}}$. Define

$$
\phi(t) \triangleq \sup _{0 \leq \tau \leq t}\|\tilde{s}(\tau)\|_{M}
$$

It follows that from (25) and (26):

$$
\|\tilde{q}(t)\| \leq\|\tilde{q}(0)\|+\frac{\sup _{0 \leq \tau \leq t}\|\tilde{s}(\tau)\|}{\lambda} \leq\|\tilde{q}(0)\|+\frac{\phi(t)}{\lambda \sqrt{k_{\underline{m}}}} \triangleq k_{e} .
$$

Note from the definition of $\tilde{s}$ that $\tilde{s}=\dot{\tilde{q}}+\lambda \tilde{q}$, we have that

$$
\begin{aligned}
& \|\dot{\tilde{q}}(t)\|=\|\tilde{s}(t)-\lambda \tilde{q}(t)\| \leq \frac{\phi(t)}{\sqrt{k_{\underline{m}}}}+\lambda k_{e} \triangleq k_{\dot{e}}, \\
& \|\dot{q}(t)\|=\left\|\dot{\tilde{q}}(t)+\mathbf{1}_{n} \otimes \dot{q}_{0}(t)\right\| \leq k_{\dot{e}}+k_{v} .
\end{aligned}
$$

Substituting (27)-(29) into (23), it follows that:

$$
\begin{aligned}
\|\Delta\| & \leq k_{\bar{m}}\left(k_{a}+\lambda k_{\dot{e}}\right)+k_{C}\left(k_{v}+\lambda k_{e}\right)\left(k_{\dot{e}}+k_{v}\right)+k_{g} \\
& =a \phi^{2}(t)+b \phi(t)+c \triangleq \gamma(t)
\end{aligned}
$$

where $a \triangleq 2 k_{C} / k_{\underline{m}}, b \triangleq\left(3 k_{C} k_{v}+2 \lambda k_{\bar{m}}+3 \lambda k_{C}\|\tilde{q}(0)\|\right) / \sqrt{k_{\underline{m}}}$, $c \triangleq k_{\bar{m}} k_{a}+k_{g}+k_{C} k_{v}^{2}+\left(2 \lambda k_{C} k_{v}+\lambda^{2} k_{\bar{m}}\right)\|\tilde{q}(0)\|+\lambda^{2} k_{C}\|\tilde{q}(0)\|^{2}$. Noting that $a, b, c$ are positive constants, and $\phi(t) \geq 0$, we get that $\gamma(t)$ is monotonically increasing on $[0, \infty)$ because $\phi(t)$ is monotonically increasing on $[0, \infty)$. If there exists some bounded disturbance in (1), with an addition of a constant in $c$, the following results still hold. Thus, the coordinated tracking algorithm (21) is robust to bounded disturbance.

Consider the following Lyapunov function candidate:

$$
V=\frac{1}{2} \tilde{s}^{T} M(q) \tilde{s} .
$$

The set-valued Lie derivative of $V$ along (22) is

$$
\begin{aligned}
& \dot{\widetilde{V}}=\mathcal{K}\left[\tilde { s } ^ { T } \left[-C(q, \dot{q}) \tilde{s}-\beta\left(H_{A} \otimes I_{p}\right) \operatorname{sgn}\left[\left(H_{A} \otimes I_{p}\right) \tilde{s}\right]\right.\right. \\
& \left.\left.-\alpha\left(H_{A} \otimes I_{p}\right) \tilde{s}+\Delta\right]+\frac{1}{2} \tilde{s}^{T} \dot{M}(q) \tilde{s}\right] \\
& =\mathcal{K}\left[\tilde{s}^{T} \beta\left(H_{A} \otimes I_{p}\right) \operatorname{sgn}\left[\left(H_{A} \otimes I_{p}\right) \tilde{s}\right]\right. \\
& \left.+\tilde{s}^{T}\left[-\alpha\left(H_{A} \otimes I_{p}\right) \tilde{s}+\Delta\right]\right] \\
& =\left\{-\alpha \tilde{s}^{T}\left(H_{A} \otimes I_{p}\right) \tilde{s}-\beta\left\|\left(H_{A} \otimes I_{p}\right) \tilde{s}\right\|_{1}+\Delta^{T} \tilde{s}\right\}
\end{aligned}
$$

where we have used Assumption (A2) to obtain the second equality and the fact that $\mathcal{K}\left[x^{T} \operatorname{sgn}(x)\right]=\left\{\|x\|_{1}\right\}$ [23] to obtain the last equality. Note that the set-valued Lie derivative of $V$ is a singleton and $V$ defined 
in (31) is continuously differentiable. Let $\dot{V}$ denote the derivative of $V$. It follows from $\dot{V} \in \dot{\widetilde{V}}$ that:

$$
\begin{aligned}
\dot{V} & =-\alpha \tilde{s}^{T}\left(H_{A} \otimes I_{p}\right) \tilde{s}-\beta\left\|\left(H_{A} \otimes I_{p}\right) \tilde{s}\right\|_{1}+\Delta^{T} \tilde{s} \\
& \leq-\alpha \tilde{s}^{T}\left(H_{A} \otimes I_{p}\right) \tilde{s}-\beta\left\|\left(H_{A} \otimes I_{p}\right) \tilde{s}\right\|+\|\Delta\|\|\tilde{s}\| \\
& \leq-\alpha \tilde{s}^{T}\left(H_{A} \otimes I_{p}\right) \tilde{s}-\left[\beta \lambda_{\min }\left(H_{A}\right)-\gamma(t)\right]\|\tilde{s}\|
\end{aligned}
$$

where we have used the fact that $\|x\| \leq\|x\|_{1}$ to obtain the first inequality, and the fact that $\left\|H_{A} x\right\| \geq \lambda_{\min }\left(H_{A}\right) x, \forall x \in \mathbb{R}^{n}$, and $\|\Delta\| \leq \gamma(t)$ from (30) to obtain the last inequality.

If we can choose $\beta$ such that $\beta \lambda_{\min }\left(H_{A}\right)-\gamma(t)>0$, then we can show that $\dot{V}$ is negative definite. However, $\gamma(t)$ is a time-varying function involving $\tilde{s}(t)$, which implies that we need to know all $\tilde{s}_{i}(t)$, $i=1, \ldots, n$, at each time to find a proper $\beta$. Unfortunately, it is not possible to do so because the leader is the neighbor of only a subset of the followers. So we need the following lemma.

Lemma 4.1: If $\beta$ is chosen such that $\beta \lambda_{\min }\left(H_{A}\right)-\gamma(0)>0$, then $\beta \lambda_{\min }\left(H_{A}\right)-\gamma(t)=\beta \lambda_{\min }\left(H_{A}\right)-\gamma(0)>0$ for all $t \geq 0$, or there exists $\bar{t} \geq 0$ such that $\|\tilde{s}(t)\| \equiv 0$ for any $t \geq \bar{t}$.

Proof: Proof of Lemma 4.1 can be found in [17].

Theorem 4.2: Consider the closed-loop system (22). Suppose that $\mathcal{G}_{A}$ is undirected and in $\overline{\mathcal{G}}_{A}$ the leader has directed paths to all followers. $\left\|q_{i}(t)-q_{0}(t)\right\| \rightarrow 0$ and $\left\|\dot{q}_{i}(t)-\dot{q}_{0}(t)\right\| \rightarrow 0, i=1, \ldots, n$, exponentially as $t \rightarrow \infty$ if $\beta$ is chosen such that $\beta>\gamma(0) / \lambda_{\min }\left(H_{A}\right)$.

Proof: Because $\mathcal{G}_{A}$ is undirected and in $\overline{\mathcal{G}}_{A}$ the leader has directed paths to all followers, it follows from Lemma (2.1) that $H_{A}$ is symmetric positive definite. Because $\beta>\gamma(0) / \lambda_{\min }\left(H_{A}\right)$, it follows from Lemma 4.1 that either $\beta \lambda_{\min }\left(H_{A}\right)-\gamma(t)=\beta \lambda_{\min }\left(H_{A}\right)-$ $\gamma(0)>0$ or there exists $\bar{t} \geq 0$ such that $\|\tilde{s}(t)\| \equiv 0$ for all $t \geq \bar{t}$. In the first case, consider the Lyapunov function candidate given by (31). Noting that $V=1 / 2\|\tilde{s}\|_{M}^{2}$, it follows from (34) that:

$$
\begin{aligned}
\dot{V} & \leq-\left[\beta \lambda_{\min }\left(H_{A}\right)-\gamma(t)\right]\|\tilde{s}\| \\
& \leq-\left[\beta \lambda_{\min }\left(H_{A}\right)-\gamma(0)\right] \frac{\|\tilde{s}\|_{M}}{\sqrt{k_{\bar{m}}}}=-\eta \sqrt{V}
\end{aligned}
$$

where $\eta \triangleq \sqrt{2 / k_{\bar{m}}}\left[\beta \lambda_{\min }\left(H_{A}\right)-\gamma(0)\right]$, and we have used the fact that $\|s\| \geq\|s\|_{M} / \sqrt{k_{\bar{m}}}$ to obtain the second inequality. After some manipulation, we get that $2 \sqrt{V(t)} \leq 2 \sqrt{V(0)}-\eta t$. Therefore, we have $V(t) \equiv 0$ and equivalently $\|\tilde{s}(t)\| \equiv 0$ when $\bar{t} \geq(2 \sqrt{V(0)}) / \eta$. In the second case, there exists $\bar{t} \geq 0$ such that $\tilde{s}(t) \equiv 0$ when $t \geq \bar{t}$. Combining both cases, we can get that $\|\tilde{s}(t)\| \equiv 0$ when $t \geq T_{1} \triangleq$ $\max \{(2 \sqrt{V(0)}\} / \eta, \bar{t}\}$, which implies that $\dot{\tilde{q}}(t)+\lambda \tilde{q}(t) \equiv \mathbf{0}_{n p}, i=$ $1, \ldots, n$, when $t \geq T_{1}$. Noting that the solution of $\dot{\tilde{q}}(t)+\lambda \tilde{q}(t) \equiv \mathbf{0}_{n p}$ is $\tilde{q}(t)=e^{-\lambda\left(t-T_{1}\right)} \tilde{q}\left(T_{1}\right)$ and $\dot{\tilde{q}}(t)=-\lambda e^{-\lambda\left(t-T_{1}\right)} \tilde{q}\left(T_{1}\right)$, we can conclude that $q_{i}(t)-q_{0}(t) \rightarrow \mathbf{0}_{p}$ and $\dot{q}_{i}(t)-\dot{q}_{0}(t) \rightarrow \mathbf{0}_{p}$ exponentially as $t \rightarrow \infty$.

Remark 4.3: From Lemma 4.1, $\beta$ can be chosen according to $\gamma(0)$, which means that the initial values $\|\tilde{q}(0)\|$ and $\|\tilde{s}(0)\|$ should be known by each follower to compute $\beta$ even if the leader is a neighbor of only a subset of the followers. However, because only the initial value is needed, it is reasonable. Also note that the lower bound of $\beta$ might be conservative. In reality, a smaller value might be chosen. Moreover, $\beta$ can be tuned according to the performance of the whole system in practice, so the accurate knowledge of $\|\tilde{q}(0)\|$ and $\|\tilde{s}(0)\|$ might not be needed.

Remark 4.4: Note that the algorithm (21) is model-independent. The bound of $\|\Delta\|$ in (23) is dependent on the bound of $\|g(q)\|$. In practice, one might know the nominal dynamics of $g_{i}\left(q_{i}\right)$, denoted as $g_{i}^{0}\left(q_{i}\right)$. Assume that $\left\|g_{i}\left(q_{i}\right)-g_{i}^{0}\left(q_{i}\right)\right\| \leq k_{\tilde{g}}$, where $k_{\tilde{g}}$ is a known positive constant generally smaller than $k_{g}$. If we choose the control algorithm as $\check{\tau}_{i}=\tau_{i}+g_{i}^{0}\left(q_{i}\right), k_{g}$ in (23) can be replaced with a smaller parameter

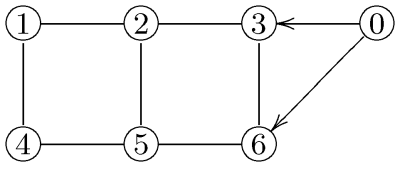

Fig. 1. Interaction graph associated with the leader and the six followers. An edge between $i$ and $j$ means that $i$ and $j$ are neighbors of each other while an arrow from 0 to $i$ means that the leader is a neighbor of follower $i$.

$k_{\tilde{g}}$. By doing so, it is no longer required that $\left\|g_{i}\left(q_{i}\right)\right\|$ is bounded. That is, Assumption (A1) can be relaxed.

Remark 4.5: From the proof in Theorem 4.2, the error vector $\tilde{s}$ will first decrease to zero in finite time. Then, $q_{i}-q_{0}$ and $\dot{q}_{i}-\dot{q}_{0}$ converge zero exponentially fast with a convergence rate $\lambda$.

Remark 4.6: In Section III, we deal with the case where the leader has a constant vector of generalized coordinate derivatives. A distributed adaptive control combined with a distributed continuous estimator is proposed. The strength of the algorithm in Section III is that the algorithm is continuous while the weakness is that the algorithm cannot be used to track a leader that has a time-varying vector of generalized coordinate derivatives when only a subset of the followers has access to the leader. In Section IV, we proposed a sliding-model control algorithm. The strength of the algorithm is that the algorithm enables tracking of a leader that has a time-varying vector of generalized coordinate derivatives under the constraint that only a subset of the followers has access to the leader. However, the weakness of the algorithm is that the algorithm is discontinuous and requires the availability of information from both the one-hop and two-hop neighbors. Therefore, both algorithms have their strengths and weaknesses and are proper under different situations.

\section{Simulation Results}

In this section, numerical simulations are preformed to show the effectiveness of the proposed control algorithms. We consider six networked two-link revolute joint arms modeled by Euler-Lagrange equations. The readers are refer to [18, pp. 259-262] for the details of the equations. In particular, the masses of links 1 and 2 of the $i$ th revolute joint arm are, respectively, $m_{1 i}=(1+0.3 i) \mathrm{kg}$ and $m_{2 i}=(1.5+0.3 i) \mathrm{kg}$, the lengths of links 1 and 2 of the $i$ th revolute joint arm are, respectively, $l_{1 i}=(0.2+0.06 i) \mathrm{m}$ and $l_{12}=(0.3+0.06 i) \mathrm{m}$, the distances from the previous joint to the center of mass of links 1 and 2 of the $i$ th revolute joint arm are, respectively, $l_{c 1 i}=(0.1+0.03 i) \mathrm{m}$ and $l_{c 2 i}=(0.15+0.03 i) \mathrm{m}$, $i=1, \ldots, 6$. The moments of inertia of links 1 and 2 are, respectively, $J_{1}=[0.0073,0.0137,0.0229,0.0355,0.0521,0.0732] \mathrm{kg} \mathrm{m}^{2}$ and $J_{2}=[0.0194,0.0309,0.0461,0.0656,0.0900,0.1198] \mathrm{kg} \mathrm{m}^{2}$. We also assume that the nominal dynamics $g_{i}^{0}\left(q_{i}\right)$ is set off from the real dynamics $g_{i}\left(q_{i}\right)$ by $10 \%$.

We assume that $\mathcal{G}_{A}$ and $\mathcal{G}_{B}$ are identical for simplicity. Fig. 1 shows the networked topology associated with the six followers and the leader. There are seven edges between the followers, and arms 3 and 6 have access to the leader (i.e., arm 0). In our simulations, we choose $a_{i j}=1, i=1, \ldots, 6, j=0, \ldots, 6$, if agent $j$ is a neighbor of agent $i$, and $a_{i j}=0$ otherwise. Let $q_{i}(0)=[(\pi / 7) i,(\pi / 8) i]^{T} \mathrm{rad}$ and $\dot{q}_{i}(0)=[0.05 i-0.2,-0.05 i+0.2]^{T} \mathrm{rad} / \mathrm{s}$, where $i=1, \ldots, 6$. For the algorithm (4), the vector of joint angles of the leader are chosen as $q_{0}(t)=[0.04 t, 0.05 t]^{T} \mathrm{rad}$, and the vector of joint angle derivatives of the leader is hence $\dot{q}_{0}=[0.04,0.05]^{T} \mathrm{rad} / \mathrm{s}$. The control parameters in (4) are chosen as $K_{i}=3 I_{2}, \alpha=1, \eta=2$, and $\Lambda_{i}=2 I_{2}$. For the algorithm (21), the vector of joint angles of the leader is chosen as $q_{0}(t)=[\cos ((2 \pi / 60) t), \sin ((2 \pi / 60) t)]^{T} \mathrm{rad}$, the vector of joint angle derivatives of the leader is hence 

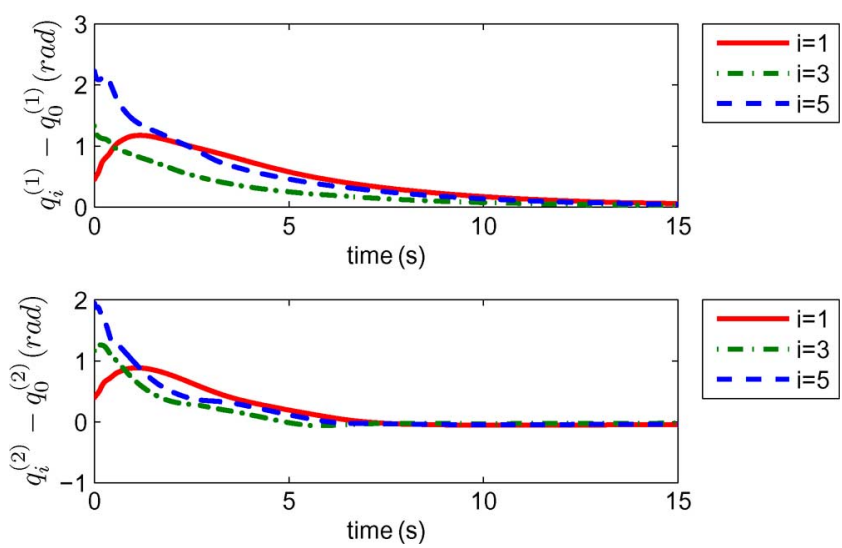

Fig. 2. Differences between the joint angles of arms 1,3, and 5 and the leader using (4).
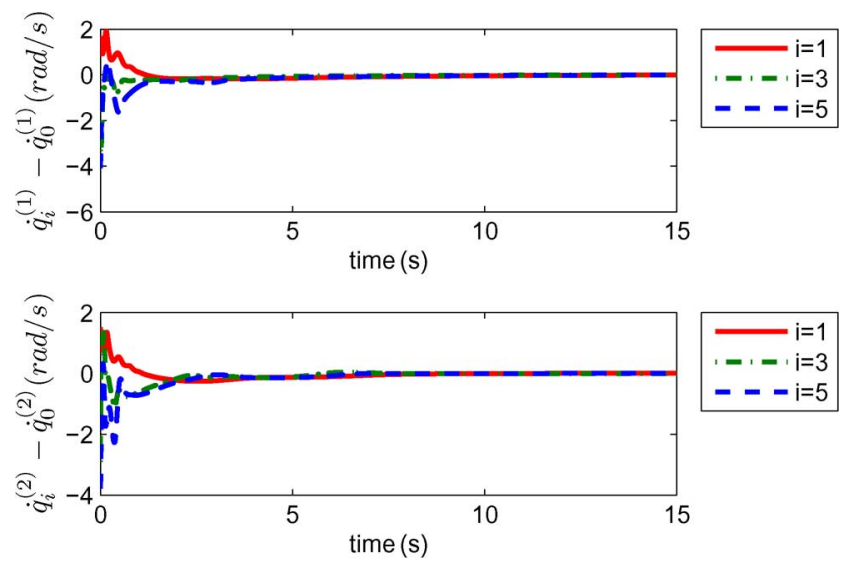

Fig. 3. Differences between the joint angle derivatives of arms 1, 3, and 5 and the leader using (4).
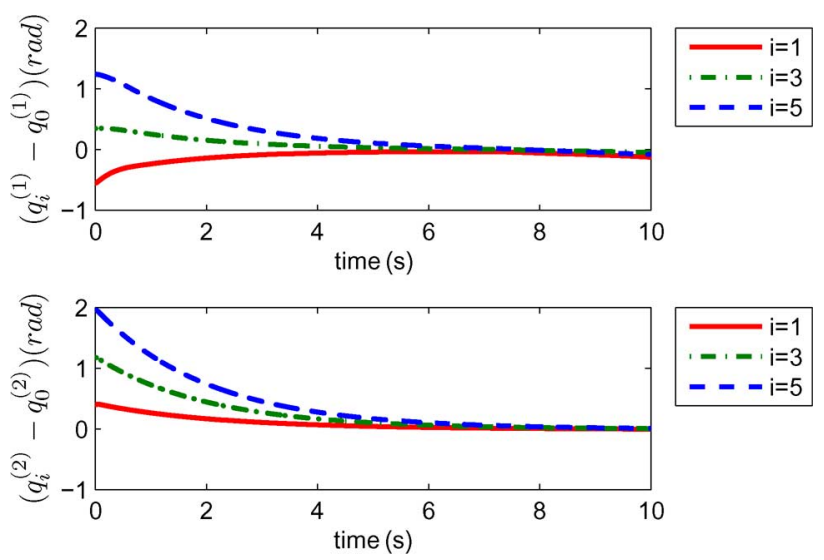

Fig. 4. Differences between the joint angle derivatives of arms 1, 3, and 5 and the leader using (21) with compensation of the nominal dynamics $g_{i}^{0}\left(q_{i}\right)$.

$\dot{q}_{0}(t)=(2 \pi / 60)[-\sin ((2 \pi / 60) t), \cos ((2 \pi / 60) t)]^{T} \mathrm{rad} / \mathrm{s}$, and the control parameters are chosen as $\alpha=5, \lambda=0.5$, and $\beta=8.5$. We use a superscript $(j)$ to denote the $j$ th component of a vector.

Fig. 2 shows the differences between the joint angles of arms 1, 3 and 5 and the leader using (4). Fig. 3 shows the differences between the joint angle derivatives of arms 1, 3 and 5 and the leader using (4). All followers' joint angles and joint angle derivatives approach those of the leader.
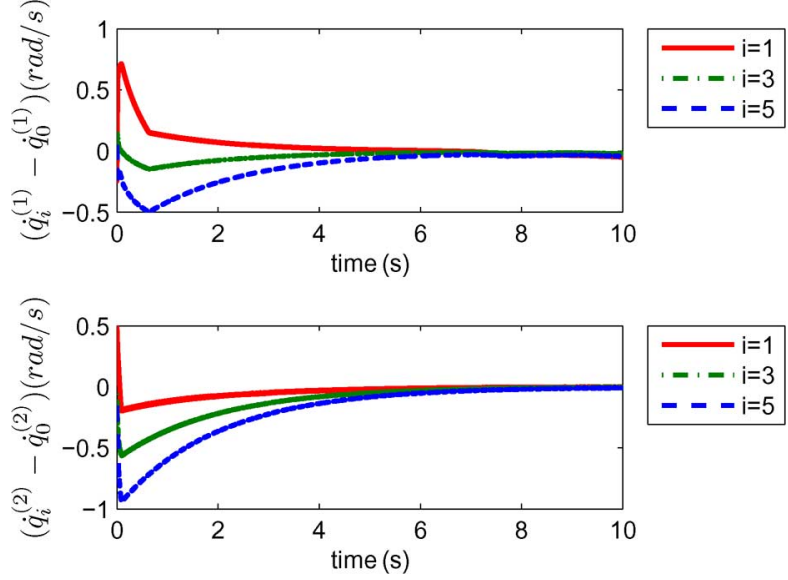

Fig. 5. Differences between the joint angle derivatives of arms 1, 3, and 5 and the leader using (21) with compensation of the nominal dynamics $g_{i}^{0}\left(q_{i}\right)$.

Fig. 4 shows the differences between the followers' joint angles of arms 1,3 , and 5 and the leader while Fig. 5 shows the differences between the joint angle derivatives of arms 1, 3, and 5 and the leader using (21) with compensation of the nominal dynamics $g_{i}^{0}\left(q_{i}\right)$. Again, all followers' joint angles and joint angle derivatives approach those of the leader.

\section{CONCLUSION}

We have studied the issues associated with distributed coordinated tracking for multiple networked Euler-Lagrange systems where only a subset of the followers has access to the leader. In the case where the leader has a constant vector of generalized coordinate derivatives, we have proposed a distributed adaptive control algorithm to account for parametric uncertainties. A distributed continuous estimator is designed to estimate the leader's vector of generalized coordinate derivatives because it is known by only a subset of the followers. In the case where the leader has a time-varying vector of generalized coordinate derivatives, we have proposed a distributed model-independent tracking controller via a sliding mode approach. The algorithm needs the information of both the neighbors and the neighbors' neighbors. The error vectors converge to a sliding surface in finite time, and then converge to zero exponentially. The control algorithm is robust to bounded disturbance.

\section{REFERENCES}

[1] W. Ren and R. W. Beard, Distributed Consensus in Multi-Vehicle Cooperative Control. London, U.K.: Springer-Verilag, 2008.

[2] Z. Qu, Cooperative Control of Dynamical Systems. London, U.K.: Springer-Verilag, 2009.

[3] F. Bullo, J. Cortes, and S. Martinez, Distributed Control of Robotic Networks. Princeton, NJ: Princeton Univ. Press, 2009.

[4] W. Ren, R. W. Beard, and E. M. Atkins, "Information consensus in multivehicle cooperative control," IEEE Control Syst. Mag., vol. 27, no. 2, pp. 71-82, Apr. 2007.

[5] R. Olfati-Saber, J. A. Fax, and R. M. Murray, "Consensus and cooperation in networked multi-agent systems," Proc. IEEE, vol. 95, no. 1, pp. 215-233, Jan. 2007.

[6] Y. Hong, J. Hu, and L. Gao, "Tracking control for multi-agent consensus with an active leader and variable topology," Automatica, vol. 42, no. 7, pp. 1177-1182, July 2006.

[7] W. Ren, "Consensus tracking under directed interaction topologies: Algorithms and experiments," IEEE Trans. Control Syst. Technol., vol. 18, no. 1, pp. 230-237, Jan. 2010.

[8] K. Peng and Y. Yang, "Leader-following consensus problem with a varying-velocity leader and time-varying delays," Physica A, vol. 388, no. 2-3, pp. 193-208, Jan. 2009. 
[9] Y. Cao and W. Ren, "Distributed coordinated tracking with reduced interaction via a variable structure approach," IEEE Trans. Autom. Control, vol. 56, no. 12, Dec. 2011, to be published.

[10] W. Ren, "Distributed leaderless consensus algorithms for networked Euler-Lagrange systems," Int. J. Control, vol. 82, no. 11, pp. 2137-2149, 2009.

[11] N. Chopra, D. M. Stipanovic, and M. W. Spong, "On synchronization and collision avoidance for mechanical systems," in Proc. Amer. Control Conf., Seattle, WA, Jun. 2008, pp. 3713-3718.

[12] D. Sun, X. Zhao, and G. Feng, "A model-free cross-coupled control for position synchronization of multi-axis motions: Theory and experiments," IEEE Trans. Control Syst. Technol., vol. 15, no. 2, pp. 306-314, Mar. 2007.

[13] M. W. Spong and N. Chopra, "Synchronization of networked Lagrangian systems," in Lagrangian and Hamiltonian Methods for Nonlinear Control, ser. Lecture Notes in Control and Information Sciences, F. Bullo and K. Fujimoto, Eds. New York: Springer-Verlag, 2007, pp. 47-59.

[14] S.-J. Chung and J.-J. E. Slotine, "Cooperative robot control and concurrent synchronization of Lagrangian systems," IEEE Trans. Robotics, vol. 25, no. 3, pp. 686-700, Jun. 2009.

[15] C. C. Cheah, S. P. Hou, and J. J. E. Slotine, "Region-based shape control for a warm of robots," Automatica, vol. 45, no. 10, pp. 2406-2411, Oct. 2009.

[16] P. F. Hokayem, D. M. Stipanovic, and M. W. Spong, "Semiautonomous control of multiple networked Lagrangian systems," Int. J. Robust Nonlin. Control, vol. 19, no. 18, pp. 2040-2055, 2009.

[17] J. Mei, W. Ren, and G. Ma, "Distributed coordinated tracking for multiple Euler-Lagrange systems," in Proc. IEEE Conf. Decision Control, Atlanta, GA, Dec. 2010, pp. 3208-3213.

[18] M. W. Spong, S. Hutchinson, and M. Vidyasagar, Robot Modeling and Control. New York: Wiley, 2006.

[19] E. Panteley and A. Loria, "Growth rate conditions for uniform asymptotic stability of cascaded time-varying systems," Automatica, vol. 37, no. 3, pp. 453-460, 2001.

[20] J.-J. E. Slotine and W. Li, Applied Nonlinear Control. Englewood Cliffs, NJ: Prentice-Hall, 1991.

[21] A. F. Filippov, Differential Equations With Discontinuous Righthand Sides. Amsterdam, The Netherlands: Kluwer, 1988.

[22] J. Cortes, "Discontinuous dynamical systems: A tutorial on solutions, nonsmooth analysis, and stability," IEEE Control Syst. Mag., vol. 28, no. 3, pp. 36-73, Jun. 2008.

[23] B. Paden and S. S. Sastry, "A calculus for computing filippov's differential inclusion with application to the variable structure control of robot manipulators," IEEE Trans. Circutis Syst., vol. CS-34, no. 1, pp. 73-82, Jan. 1987.

\section{Linear Input-Output Equivalence and Row Reducedness of Discrete-Time Nonlinear Systems}

\author{
Ülle Kotta, Member, IEEE, Zbigniew Bartosiewicz, \\ Sven Nõmm, and Ewa Pawłuszewicz
}

\begin{abstract}
The problem of linear input-output (i/o) equivalence of meromorphic nonlinear control systems, described by implicit higher order difference equations, is studied. It is proved that any system is linearly $i / 0$ equivalent to a row-reduced form. The constructive algorithm is given for finding the required transformation. The latter amounts to 1) multiply the set of i/o equations $\varphi=0$ from left by a unimodular matrix $A(\delta)$, whose entries are non-commutative polynomials in the forward-shift operator $\delta$, and 2) define certain multiplicative subset of the difference ring of analytic functions which introduces some inequations that should be satisfied.
\end{abstract}

Index Terms-Discrete-time systems, input-output (i/o) models, linear input-output ( $\mathrm{i} / \mathrm{o})$ equivalence transformations, meromorphic nonlinear control systems, polynomial approach.

\section{INTRODUCTION}

One of the central themes in the system theory is the problem of representing a system in a form that is convenient for the particular purpose and of transforming one representation into another. Particularly, for linear systems, it is well-known that an arbitrary set of higher order input-output (i/o) difference equations can be always transformed into an i/o equivalent set of equations, having a row-reduced form [2], [20].

The main purpose of this technical note is to introduce and characterize the linear i/o equivalence for nonlinear control system, described by the set of meromorphic higher order i/o difference equations, and to transform the set of equations via linear i/o equivalence transformation into an equivalent set of equations in row-reduced form using the polynomial approach. Our equivalence transformation does not change the zeros (solutions) of the set of i/o equations. Note that for linear time-invariant systems, under the additional assumption that two sets have the same number of equations, linear i/o equivalence coincides with the i/o equivalence as defined in the paper [17]. ${ }^{1}$ Our interest in row-reduced form originates from the fact that this is a necessary step for realization of the $i / o$ difference equations in the classical state space form. Note that the realization procedure in [16] as well as its extension to the MIMO case requires the system equations to be given in the explicit form, corresponding to the Popov canonical form in the linear case. This form defines explicitly the minimal set of independent system variables and allows to compute explicitly the dependent variables. Once the set of nonlinear higher order difference equations is in the row- and column-reduced form, it is extremely easy to transform these equations into the Popov form. So, the row- and column-reduced

Manuscript received November 30, 2009; revised April 21, 2010, and September 15, 2010; accepted January 24, 2011. Date of publication February 07, 2011; date of current version June 08, 2011. This work was supported by Estonian Science Foundation Grant 6922 and by the Białystok University of Technology Grant W/WI/1/2010. Recommended by Associate Editor P. Tsiotras.

Ü. Kotta and S. Nõmm are with the Control Systems Department, Institute of Cybernetics, Tallinn University of Technology, Tallinn 12618, Estonia (e-mail: \{kotta, sven\}@cc.ioc.ee)

Z. Bartosiewicz and E. Pawłuszewicz are with Białystok University of Technology, Białystok 15-351, Poland (e-mail: \{bartos, epaw\}@pb.edu.pl).

Digital Object Identifier 10.1109/TAC.2011.2112430

${ }^{1}$ This is not the same notion of $\mathrm{i} / \mathrm{o}$ equivalence that is considered in [4]. The latter is called, according to [17], the transfer equivalence, and may change the solutions of the set of i/o equations. 